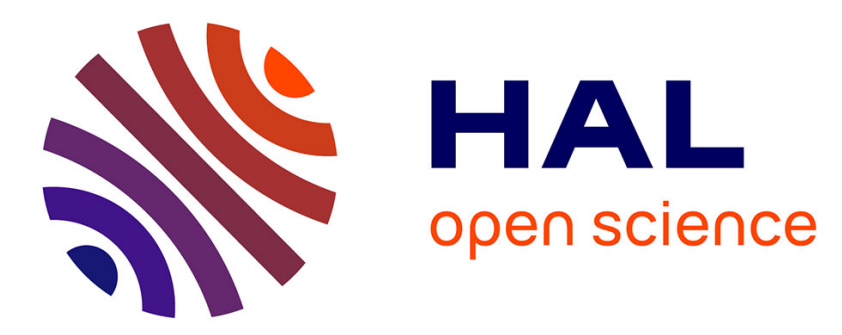

\title{
Experimental and numerical investigation of energy dissipation in elastomeric rotational joint under harmonic loading
}

Hanen Jrad, Jean-Luc Dion, Franck Renaud, Imad Tawfiq, Mohamed Haddar

\section{- To cite this version:}

Hanen Jrad, Jean-Luc Dion, Franck Renaud, Imad Tawfiq, Mohamed Haddar. Experimental and numerical investigation of energy dissipation in elastomeric rotational joint under harmonic loading. Mechanics of Time-Dependent Materials, 2016, 21 (2), pp.177-198. 10.1007/s11043-016-9325-9 . hal01534458

\author{
HAL Id: hal-01534458 \\ https://hal.science/hal-01534458
}

Submitted on 24 Nov 2021

HAL is a multi-disciplinary open access archive for the deposit and dissemination of scientific research documents, whether they are published or not. The documents may come from teaching and research institutions in France or abroad, or from public or private research centers.
L'archive ouverte pluridisciplinaire HAL, est destinée au dépôt et à la diffusion de documents scientifiques de niveau recherche, publiés ou non, émanant des établissements d'enseignement et de recherche français ou étrangers, des laboratoires publics ou privés.

\section{(ㄷ)(1) $\$$}

Distributed under a Creative Commons Attribution - NonCommerciall 4.0 International 


\title{
Experimental and numerical investigation of energy dissipation in elastomeric rotational joint under harmonic loading
}

\author{
Hanen Jrad ${ }^{1,2}$ • Jean Luc Dion ${ }^{1}$. Franck Renaud ${ }^{1}$. \\ Imad Tawfiq ${ }^{1} \cdot$ Mohamed Haddar ${ }^{2}$
}

\begin{abstract}
This paper focuses on energy losses caused by inner damping and friction in an elastomeric rotational joint. A description of the design of a new experimental device intended to characterize dynamic stiffness in rotational elastomeric joint is presented. An original method based on Lagrange's equations, which allows accurately measuring forces and torques only with accelerometers, is proposed in order to identify dissipated energy in the rotational elastomeric joint. A rheological model developed taking into account dependence of the torque and the angular displacement (rotation). Experimental results and simulations used to quantify the dissipated energy in order to evaluate the damping ratio are presented and discussed.
\end{abstract}

Keywords Vibratory energy dissipation - Nonlinear dynamic behavior · Dynamic material tests

H. Jrad

hanen.j@gmail.com

J.L. Dion

jean-luc.dion@supmeca.fr

F. Renaud

franck.renaud@supmeca.fr

I. Tawfiq

imad.tawfiq@supmeca.fr

M. Haddar

mohamed.haddar@enis.rnu.tn

1 Laboratoire d'Ingénierie des Systèmes Mécaniques et des Matériaux (LISMMA), Institut Supérieur de Mécanique de Paris, 3 rue Fernand Hainaut, 93407 Saint Ouen Cedex, Paris, France

2 Laboratoire de Modélisation, Mécanique et Productique (LA2MP), Ecole Nationale d'Ingénieurs de Sfax, BP N 1173, Sfax, 3038, Tunisia 


\section{Introduction}

Elastomeric materials are often integrated into mechanical structures in order to minimize vibrations and for sealing functions. Elastomeric components are indispensable elements in designing intended dynamic behavior of mechanical systems. However, elastomeric materials exhibit significant nonlinear behavior (Coveney et al. 1995) which is generally neglected or, at most, largely simplified. Consequently, to ameliorate characterization and prediction, a complete mechanism model ought to include connections and joints between solids, not to merely model the free relative degrees of freedom to consider the related kinematics but also stiffness and damping.

Over the years, much research has been dedicated to describe the nonlinear behavior of an elastomer and to determine its dynamic characteristics. A nonlinear viscoelastic solid model was developed by Gandhi and Chopra (1996) in which a nonlinear lead spring was used in series with a single linear Kelvin chain. Using this model, the variation of complex modulus with oscillation amplitude closely matched experimental data. However, parameters in this model are identified using amplitude-dependent complex modulus data. As a result, the nonlinear hysteresis behavior could not be captured using this model. Maxwell model represented by Park (2001) as a spring and dash-pot connected in series and Kelvin Voigt model which consists of a spring and dash-pot in parallel are efficient only on a small frequency range. The Zener model (see Huynh et al. 2002) underestimates the dynamic stiffness at low frequencies and overestimates it at high ones. Just like Kelvin Voigt model, the Zener model is unable to capture the frequency dependence of the phase angle.

Elastomeric joints typically demonstrate tribo-visco-elastic behavior ( $\mathrm{Hu}$ and Wereley 2007). Indeed, elastomeric components play an essential part in vibration damping and energy dissipation in mechanical systems. Energy dissipation in elastomeric joint results not only from inner damping caused by material molecular structure but also friction forces at clamping areas as joints play an important role in the energy losses.

A number of researchers have combined springs, dash-pots and frictional slides to represent tribo-visco-elastic behavior of elastomeric joints. In the model developed by Tarzanin and Panda (1995), the elastomeric behavior was represented by a nonlinear spring and a nonlinear Coulomb friction damper. This model was based on single frequency elastomer data and matched the value of energy dissipation per cycle. Panda et al. (1996) replaced the Coulomb friction damping element with a variable friction damping element whose force was calculated based on the peak displacement of excitation when the velocity was zero. This model correlated well with the experimental hysteresis data, but the effectiveness of this model over a range of amplitudes and frequencies has not been demonstrated in the literature.

To improve the description of dynamic behavior, other models have been developed with internal state variables that determine the level of friction and velocity. The evolution in time of the state variables is governed by a set of differential equations. The Dahl Model (see Dahl 1968) incorporates a state variable to model presliding displacement. Dahl's starting point was the linear stress-strain curve and steady-state version of the Dahl model is Coulomb friction. A stress shift function used by Strganac (1997) to formulate a nonlinear time domain model for elastomers, but the nonlinear integral formulation in the model was difficult to implement in traditional aeromechanical analysis. Lesieutre and Bianchini (1993) developed an anelastic displacement field (ADF) method to describe the frequency-dependent behavior of viscoelastic materials. It was based on the notion of scalar internal variables or augmenting thermodynamic fields (ATF) (Lesieutre and Mingori 1990) that described the interaction of the displacement field with irreversible processes occurring at the material level. 
An ADF based model containing nonlinear fractional derivatives and frictional elements is developed by Ramrakhyani et al. (2004). This model used eight parameters to capture the amplitude-dependent and mild frequency-dependent modulus. However, the prediction of hysteresis loops was not noticeably improved, and the determination of model parameters remains complicated.

In order to capture the characteristic nonlinear hysteretic behavior of elastomeric materials, (Cantournet et al. 2009) worked on the identification of the nonlinear behavior in large deformations and presented a thermodynamical model of internal friction and hysteresis of elastomers based on the study of physical structure of filled elastomers which allows the identification of the state variables needed to model the behavior of elastomeric materials. Almajid (2004) offered a generalization of Dahl model, which was then applied to mechanical components with nonlinear behavior especially in mechanical assemblies whose purpose is to rigidly bind two metallic parts which dissipate energy by macro-slip in interfaces. The Iwan model which is an internal variable model introduced by Segalman (2005, 2006) provides formulation of idealized "Jenkins elements" and appears capable of reproducing the important joint qualitative properties using only a small number of parameters, but this model reflects poorly viscoelasticity. Skouvaklis et al. (2012) presented a study of rubber sliding on ice, investigating speed, load, temperature, and rubber properties. Dahl model was used in this study to characterize friction of elastomeric joints.

Several experimental investigations have been carried out to characterize dynamic behavior of elastomeric components depending on frequency. Barbosa and Farage (2008) and Castello et al. (2008) used Frequency Response Function (FRF) which can only characterize the frequencies of modes and not a wide frequency band. Luo et al. (2010) and Liu and Hoo Fatt (2011) used Dynamic Mechanical Analysis (DMA) to identify mechanical proprieties of elastomeric rubber and to describe strain-amplitude-dependent hysteresis losses.

The aim of this work is to characterize the nonlinear behavior caused by vibration damping and friction and to identify energy losses of elastomeric joint under rotational harmonic loading. A new experimental setup is designed to identify the dynamic behavior of viscoelastic component; torsion dynamic tests are performed on elastomeric joint (rubber sample) for different excitation frequencies.

In this paper, after having presented a description of the experimental procedure to characterize dynamics of the elastomeric joint, dynamic properties are identified using Lagrange's equations to determine the equation of motion of the system, and a new approach of post-processing experimental signals and verification of the post processing equations in order to acquire angular accelerations from measured tangential accelerations are presented.

In Sect. 3, a rheo-tribological model is extended to take the dependence of the torque on the angular displacement into account. The capital concern of this extension subsists in the technology of rotational elastomeric joint that often exhibits such dynamic behavior induced essentially from vibration damping and interfaces friction. Parameters of the rheotribological extended model are identified from torsion dynamic tests for different excitation frequencies. Comparison between measurements and simulations of hysteretic behavior of the elastomeric joint are carried out in Sect. 4 and used to quantify the dissipated energy in order to compute the damping ratio.

\section{Characterization of dynamic stiffness under cyclic rotational loading}

One of the unique characteristics of viscoelastic elastomeric materials is that their properties are influenced by many parameters. They can include frequency, dynamic displacement rate, 
Fig. 1 Hysteresis loop for rotational harmonic excitation

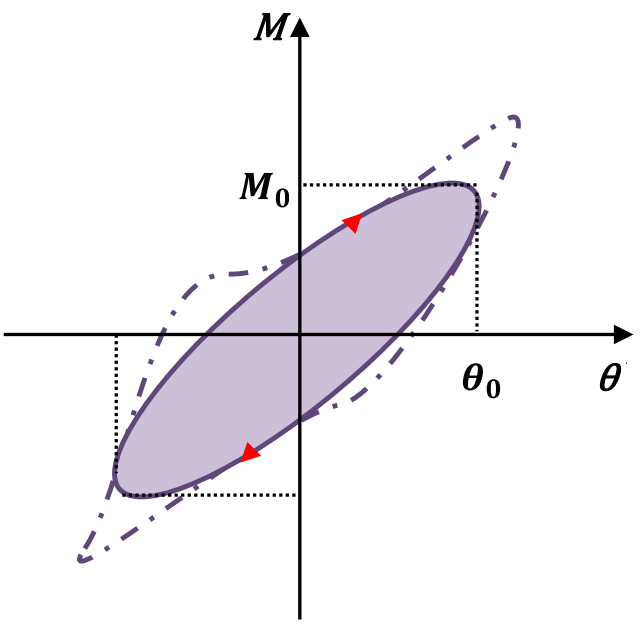

static pre-load, time effects such as creep and relaxation, and other irreversible effects. In working with this class of materials, we strive to define the materials complex modulus (stiffness and damping properties) as a function of these parameters.

Considering an elastomeric rubber sample subjected to cyclic sinusoidal angular displacement (rotation) of angular frequency $\omega$, the corresponding torque response is assumed to be sinusoidal at the same frequency but with a dephasing angle $\varphi$. However, this assumption, called the first harmonic, is not sufficient. Typically, the system response contains higher order harmonics, and the real response is expressed as follows:

$$
M(t)=\sum_{k} M_{k} \sin \left(k \omega t+\varphi_{k}\right)
$$

The elliptical shape in Fig. 1 corresponds to a typical hysteresis loop for a viscoelastic material; however, the dashed-dotted curve corresponds to a realistic nonlinear response. Under the assumption of the first harmonic, the energy dissipated as heat during a cycle corresponds to the area enclosed by the loading and unloading curves shown in Fig. 1 and expressed as follows:

$$
E_{d}=\oint M(t) d x=M_{0} \theta_{0} \omega \int_{0}^{T} \cos (\omega t) \sin (\omega t+\varphi) d t=\pi M_{0} \theta_{0} \sin (\varphi) .
$$

The dissipated energy during a hysteresis cycle reflects the damping properties of the material.

The complex modulus $K^{*}(\omega)$ relates the Fourier transform of the angular displacement $\hat{\theta}(\omega)$ and the corresponding torque $\hat{M}(\omega)$ which is defined using Hooke's Law as follows:

$$
\hat{M}(\omega)=K^{*}(\omega) \hat{\theta}(\omega) .
$$

The complex modulus is usually used to characterize viscoelastic materials under harmonic excitation. The dynamic stiffness is determined point by point and only at the excitation frequency. The torque response is a multi-harmonic-Fourier series, only the response of the torque to the fundamental frequency of the excitation is kept. 


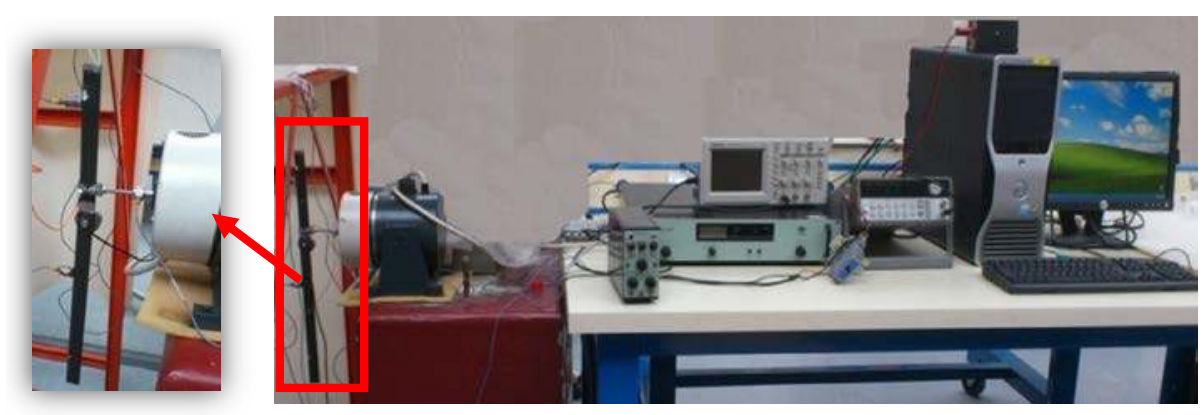

Fig. 2 Experimental device of torsion dynamic tests

The dynamic stiffness for an angular frequency $\omega_{0}$ is represented as:

$$
K^{*}(\omega)=\left.\frac{\hat{M}(\omega)}{\hat{\theta}(\omega)}\right|_{\omega=\omega_{0}}=\frac{M_{0}}{\theta_{0}} \cdot \exp (j \varphi)=K^{\prime}(\omega)+j K^{\prime \prime}(\omega),
$$

$\left|K^{*}\right|=\frac{M_{0}}{\theta_{0}}$ is the dynamic stiffness magnitude.

The storage (or in-phase) modulus is a measure of the energy stored over a cycle or period of oscillation

$$
K^{\prime}=\left|K^{*}\right| \cos \varphi=\frac{M_{0}}{\theta_{0}} \cdot \cos \varphi .
$$

The loss (or quadrature) modulus is a measure of the energy dissipated over a cycle

$$
K^{\prime \prime}=\left|K^{*}\right| \sin \varphi=\frac{M_{0}}{\theta_{0}} \cdot \sin \varphi .
$$

\section{Description of the experimental setup}

A description of the experimental device particularly designed to characterize vibrational damping causing energy dissipation by both inherent damping and interfaces friction in a rotational elastomeric joint is presented.

The experimental bench is made up of two distinct parts, see Fig. 2. An operative part, called test bench, allows applying cyclic mechanical loads to the sample. The bench is instrumented. The second part is intended to generate signals and process the data coming from the operative part to control it. This part includes all vibration analysis systems.

The test bench is composed of two rectangular sections beams mechanically coupled by a connection formed by a screw and the cylindrical rubber sample which dimensions $3 \mathrm{~mm}$ thickness and $14 \mathrm{~mm}$ diameter. The rubber sample is placed between the two rigid, flat and parallel plates at the juncture of clamping area. Fixtures and grips were designed and machined to hold the specimen in place as exposed in Fig. 3.

Mechanical cyclic loads are made up using an electrodynamic shaker as shown in the schema of Fig. 4. The electrodynamic shaker induces a vibratory motion of the first beam, the structure being free (not suspended), and under excitation a torque is created at the junction, which leads to the motion of the second beam. Harmonic sine forces are performed using the shaker. The force is measured directly with a force sensor incorporated in the 


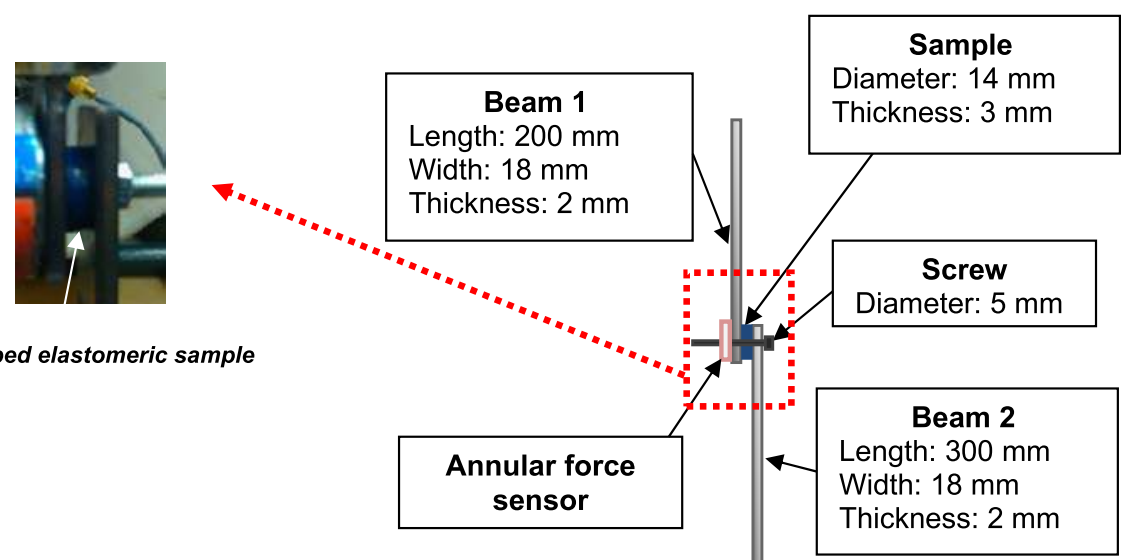

Fig. 3 Schema of vibrating mechanism

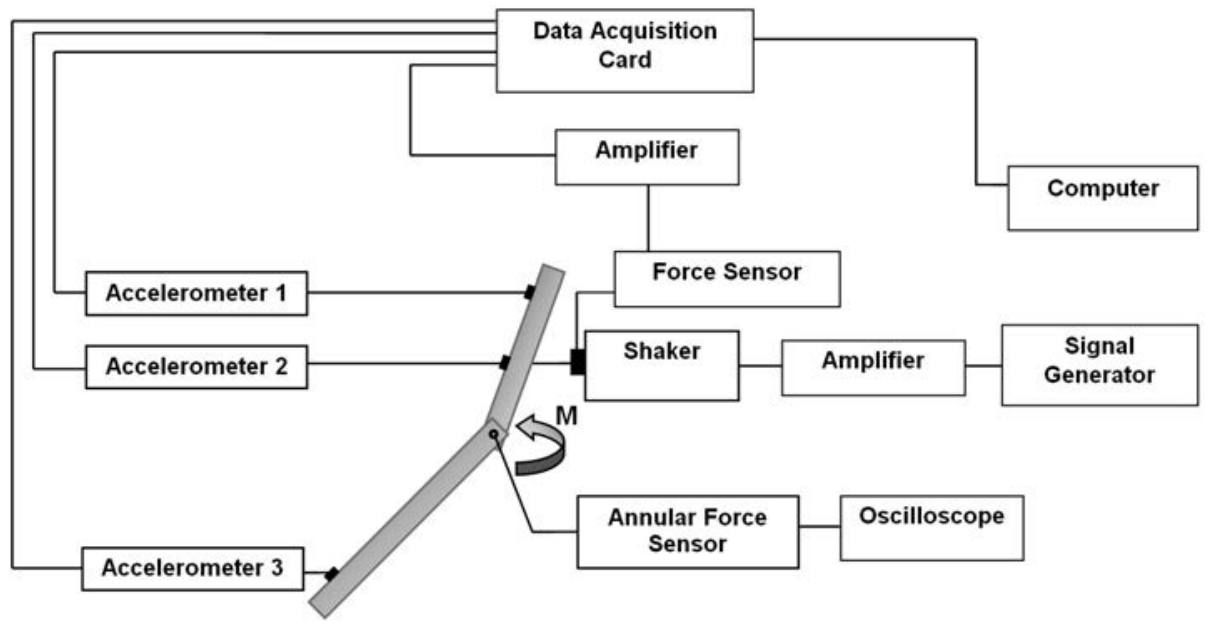

Fig. 4 Schema of the experimental setup

loading axis (between the shaker and the first beam). The force sensor is connected to an operational amplifier. This connection allows controlling the shaker force. Vibration measurements are carried out using three accelerometers placed as follows: two on the first beam and one on the second beam. A schema of the experimental setup is shown in Fig. 4.

Elastomeric sample undergoes a normal preload and twisting moment (torque). An annular force sensor is used to determine the preload during dynamic torsion tests. To determine the value of the preload at the junction, the annular force sensor is connected to an oscilloscope.

Tests are performed with sweeping sine signal created by a signal generator to power the shaker. Excitations are carried out at stabilized frequency using the signal generator. The 


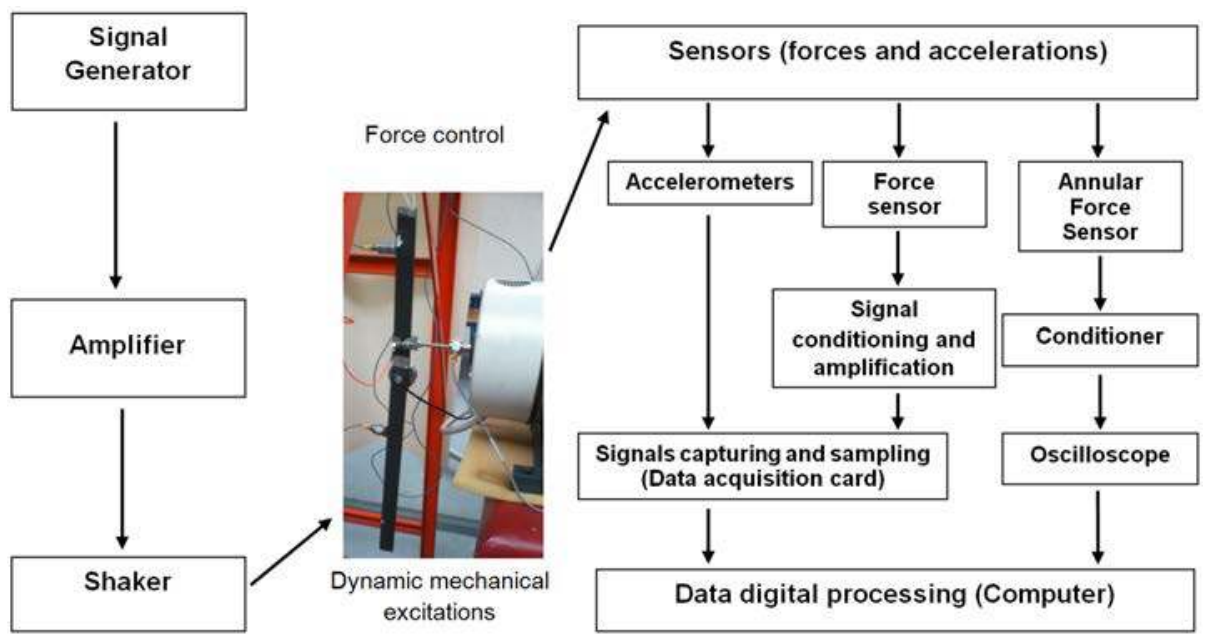

Fig. 5 Vibratory analysis chain

shaker is controlled by the amplifier and the system is managed by a spectral analyzer computer code. Figure 5 shows the vibratory analysis chain. Devices of the vibratory analysis chain are well shown in the experimental bench presented in Fig. 2.

The force signal at the junction of the two beams (preload) is filtered and captured in the oscilloscope. Preload is seen as a constant signal on the oscilloscope.

Before the acquisition of measurements, to account for stress softening effect, called the 'Mullins Effect', (Cantournet et al. 2009), the elastomeric rubber sample "is cycled" until its dynamic characteristics are identical between two consecutive readings. In general, these repeatability tests were used to check the convergence of the behavior towards stable characteristics.

For the various tests, we neglected any thermal gradient within the rubber. Testing was carried out with varying excitation amplitudes and frequencies, and all tests were conducted at room temperature: $20^{\circ} \mathrm{C}$. Excitation frequencies chosen are [5, 30, 60, 75] Hz. Elastomeric material presents different behavior according to amplitude of angular displacement (rotation) and conforming to the imposed static preload as illustrated in works of Saad et al. (2006) and Rendek and Lion (2010).

Tests are performed for different levels of static preload imposed at the juncture of clamping area $P=[45,100,150,500] \mathrm{N}$. Tangential accelerations and forces signals are transferred to a computer via the data acquisition card with a sampling frequency $f_{s}=2 \mathrm{kHz}$. The dynamic stiffness is obtained, for each frequency of excitation by the Frequency Response Function: Twisting moment (torque)/Angular displacement (rotation) identified using Lagrange's formalism.

\section{Experimental characterization}

\subsection{Experimental approach}

The aim of the experimental investigation is to evaluate the energy dissipated by both inherent damping and interfaces friction in a rotational elastomeric joint in order to evaluate the 


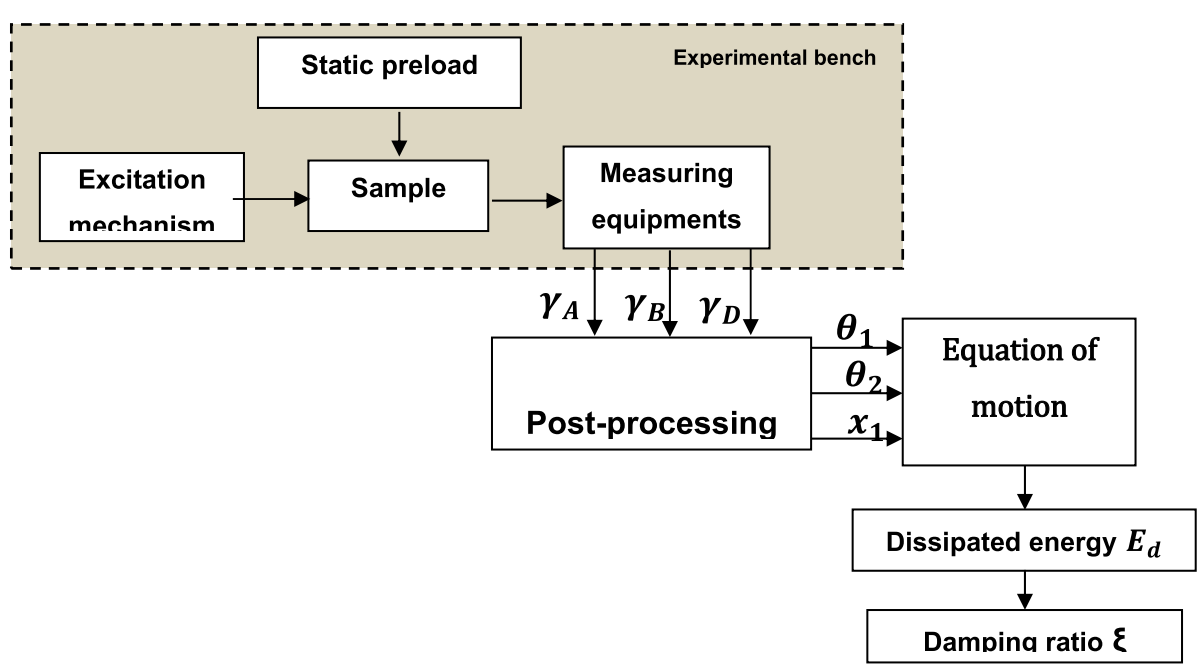

Fig. 6 Experimental methodology

damping ratio. To reach this goal, two phases are indispensable to precisely measure forces and torques only with accelerometers.

- The first phase is to find the equation of motion of the system to express the torque according to the angles of rotation (degrees of freedom of the structure).

- The second step involves post processing signals delivered by accelerometers in order to transform the measured tangential accelerations in angular accelerations and then integrated them into system motion's equations in order to obtain the hysteresis curves, i.e., the torque according to angular displacement.

Consequently, dissipated energy at elastomeric joint level is given by the closed area of hysteresis loops. Figure 6 provides better explanation of experimental methodology.

\subsection{Equation of motion assessment}

Lagrange's equations are used to find the equations of motion of the structure shown in Fig. 7.

Three independent parameters $\theta_{1}, \theta_{2}$ and $x_{1}$ are required to describe the motion of the structure.

\subsubsection{Kinetic energy of the system}

The kinetic energy of the system is due to tangential and angular displacement; it is expressed as a function of parameters of the structure as follows:

$$
\begin{aligned}
E_{c}= & \frac{1}{2}\left(m_{1}+m_{2}\right) \dot{x}_{1}^{2}+\left[\frac{I_{1}}{2}+\frac{m_{2}}{2}\left(\frac{l_{1}}{2}-e\right)^{2}\right] \dot{\theta}_{1}^{2}+\left[\frac{I_{2}}{2}+\frac{m_{2}}{2}\left(\frac{l_{2}}{2}-e\right)^{2}\right] \dot{\theta}_{2}^{2} \\
& +m_{2} \dot{x}_{1} \dot{\theta}_{1}\left(\frac{l_{1}}{2}-e\right) \cos \theta_{1}+m_{2} \dot{x}_{1} \dot{\theta}_{2}\left(\frac{l_{2}}{2}-e\right) \cos \theta_{2}
\end{aligned}
$$


Fig. 7 Schema of motion of the two beams

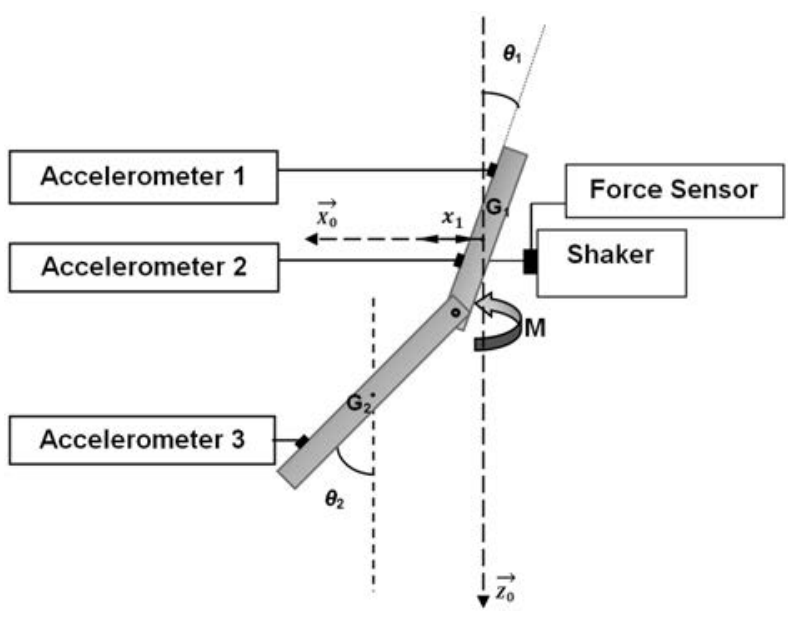

$$
+m_{2}\left(\frac{l_{2}}{2}-e\right)\left(\frac{l_{1}}{2}-e\right) \dot{\theta}_{1} \dot{\theta}_{2} \cos \left(\theta_{1}-\theta_{2}\right),
$$

where

$m_{i}$, mass of beam $i(i=1,2)$;

$I_{i}$, inertia moment of beam $i(i=1,2)$;

$l_{i}$, length of beam $i(i=1,2)$;

$\theta_{i}$, angular displacement of beam $i(i=1,2)$;

$x_{i}$, tangential displacement of center of gravity $G_{i}$ of beam $i(i=1,2)$;

$e$, length of common surface at junction between the two beams as shown in Fig. 8 .

\subsubsection{Potential energy of the system}

The potential energy of the system results from gravity, it is expressed as

$$
E_{p}=m_{2} g\left[\left(\frac{l_{2}}{2}-e\right)\left(1-\cos \theta_{2}\right)+\left(\frac{l_{1}}{2}-e\right)\left(1-\cos \theta_{1}\right)\right]
$$

where $g$ is the gravitational acceleration.

\subsubsection{Lagrange's equations}

The Lagrange equations are given by the following system:

$$
\left\{\begin{array}{l}
\frac{d}{d t} \frac{\partial E_{c}}{\partial \dot{\theta}_{1}}-\frac{\partial E_{c}}{\partial \theta_{1}}+\frac{\partial E_{p}}{\partial \theta_{1}}=M_{F} \\
\frac{d}{d t} \frac{\partial E_{c}}{\partial \dot{\theta}_{2}}-\frac{\partial E_{c}}{\partial \theta_{2}}+\frac{\partial E_{p}}{\partial \theta_{2}}=M \\
\frac{d}{d t} \frac{\partial E_{c}}{\partial \dot{x}_{1}}-\frac{\partial E_{c}}{\partial x_{1}}+\frac{\partial E_{p}}{\partial x_{1}}=F
\end{array}\right.
$$

where 
Fig. 8 Diagram of accelerometers positions on the two beams

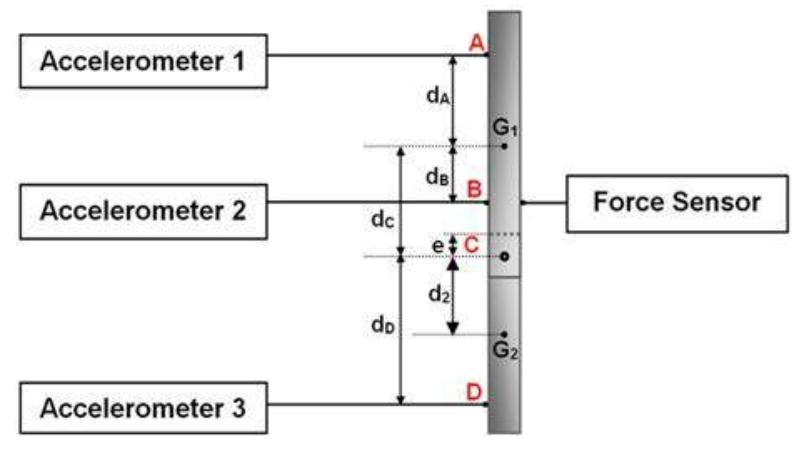

$F$, the driving force of the shaker;

$M$, the twisting moment (torque) at the junction between the two beams;

$M_{F}$, the moment of the force $F$.

Considering $d_{1}=\frac{l_{1}}{2}-e$ and $d_{2}=\frac{l_{2}}{2}-e$, after derivation, we find the expressions of $M_{F}, F$ and $M$ :

$$
\left\{\begin{aligned}
M_{F}= & \left(I_{1}+m_{2} d_{1}^{2}\right) \ddot{\theta}_{1}+m_{2} d_{1} \ddot{x}_{1} \cos \theta_{1}+m_{2} d_{1} d_{2} \ddot{\theta}_{2} \cos \left(\theta_{1}-\theta_{2}\right)+m_{2} d_{1} d_{2} \dot{\theta}_{2}^{2} \sin \left(\theta_{1}-\theta_{2}\right) \\
& +m_{2} g d_{1} \sin \theta_{1} \\
M= & \left(I_{2}+m_{2} d_{2}^{2}\right) \ddot{\theta}_{2}+m_{2} d_{2} \ddot{x}_{1} \cos \theta_{2}+m_{2} d_{1} d_{2} \ddot{\theta}_{1} \cos \left(\theta_{1}-\theta_{2}\right)-m_{2} d_{1} d_{2} \dot{\theta}_{1}^{2} \sin \left(\theta_{1}-\theta_{2}\right) \\
& \quad+m_{2} g d_{2} \sin \theta_{2} \\
F= & \left(m_{1}+m_{2}\right) \ddot{x}_{1}+m_{2} d_{2} \ddot{\theta}_{2} \cos \theta_{2}-m_{2} d_{2} \dot{\theta}_{2}^{2} \sin \theta_{2}+m_{2} d_{1} \ddot{\theta}_{1} \cos \theta_{1}-m_{2} d_{1} \dot{\theta}_{1}^{2} \sin \theta_{1}
\end{aligned}\right.
$$

In what follows, we are interested in using the expression of twisting moment as a function of rotation angles.

$$
\begin{aligned}
M= & \left(I_{2}+m_{2} d_{2}^{2}\right) \ddot{\theta}_{2}+m_{2} d_{2} \ddot{x}_{1} \cos \theta_{2}+m_{2} d_{1} d_{2} \ddot{\theta}_{1} \cos \left(\theta_{1}-\theta_{2}\right)-m_{2} d_{1} d_{2} \dot{\theta}_{1}^{2} \sin \left(\theta_{1}-\theta_{2}\right) \\
& +m_{2} g d_{2} \sin \theta_{2} .
\end{aligned}
$$

\subsection{Post processing of experimental data}

In this part, we try to find expressions of tangential accelerations $\ddot{x}_{1}, \ddot{x}_{2}$ and angular accelerations $\ddot{\theta}_{1}, \ddot{\theta}_{2}$ of beam 1 and beam 2 , respectively, from signals delivered by accelerometers. Figure 8 shows a diagram of the positions of accelerometers on the structure.

Measured accelerations at the points A, B and D $\left(\gamma_{A}, \gamma_{B}\right.$ and $\left.\gamma_{D}\right)$ are expressed according to the structure parameters. Accelerations $\gamma_{A}$ and $\gamma_{B}$ are given by:

$$
\begin{aligned}
& \gamma_{A}=\ddot{x}_{1}+d_{A} \ddot{\theta}_{1}, \\
& \gamma_{B}=\ddot{x}_{1}+d_{B} \ddot{\theta}_{1}, \\
& \gamma_{D}=\ddot{x}_{2}+d_{D} \ddot{\theta}_{2} .
\end{aligned}
$$

Then, we obtain tangential acceleration and angular acceleration at the center of gravity $G_{1}$ of the beam 1:

$$
\ddot{\theta}_{1}=\frac{\gamma_{A}-\gamma_{B}}{d_{A}-d_{B}}
$$




$$
\ddot{x}_{1}=\frac{1}{2}\left(\gamma_{A}+\gamma_{B}-\left(d_{A}+d_{B}\right) \ddot{\theta}_{1}\right) \text {. }
$$

The point $\mathrm{C}$ is at the junction between the two beams; therefore, the acceleration $\gamma_{c}$ can be expressed in two ways:

$$
\begin{aligned}
& \gamma_{c \in 1}=\ddot{x}_{1}+d_{C} \ddot{\theta}_{1}, \\
& \gamma_{c \in 2}=\ddot{x}_{2}-d_{2} \ddot{\theta}_{2} .
\end{aligned}
$$

Thus, the tangential acceleration and angular acceleration at the center of gravity $G_{2}$ of the beam 2 are expressed as:

$$
\begin{aligned}
& \ddot{\theta}_{2}=\frac{\gamma_{D}-\gamma_{c \in 1}}{\left(d_{D}+d_{2}\right)}, \\
& \ddot{x}_{2}=\frac{1}{2}\left(\gamma_{c \in 2}+\gamma_{D}-\left(d_{D}-d_{2}\right) \ddot{\theta}_{2}\right) .
\end{aligned}
$$

In order to obtain displacements, the first and second integration of acceleration signals are necessary. The Fourier transform (FFT) and the inverse Fourier transform (IFFT) are used in the integration algorithm in order to reduce the noise of acceleration signals and to reconstruct the input accelerations.

Assuming that $\theta=\theta_{2}-\theta_{1}$, which is the angular displacement (rotation), the equation of motion $M=f(\theta)$ can be subsequently determined. Figure 9 provides a better explanation of post-processing methodology.

\subsection{Experimental results}

Twisting moment (torque) and angular displacement (rotation) relationships are plotted using signals delivered by accelerometers. Hysteresis loops obtained from the experimental results of torque versus angular displacement at an excitation frequency of $60 \mathrm{~Hz}$ and a preload imposed at the juncture of clamping area $P=100 \mathrm{~N}$ are presented in Fig. 10.

It can be seen that at low amplitudes, the experimental hysteresis plots are nearly elliptical in shape, while for higher amplitudes there are deviations from this elliptical behavior. These deviations are due to the viscoelastic and frictional damping.

Depending on the preload imposed to the sample, nonlinear dynamical effects due to friction can be expected at the juncture of the clamping area of contact of the beams. For small displacements, friction is driven by micro-slip and partial slip while for high displacement the shape of the curve clearly shows macro slipping behavior.

The area inside the curve is the dissipated energy as heat during micro-slip per cycle.

The more is the hysteresis in torque-angular displacement areas: the greater the energy dissipation, the higher the damping ability.

\section{Nonlinear rheo-tribological model}

\subsection{Modeling approach}

Vibration damping is a phenomenon by which mechanical energy is dissipated and mostly converted to heat energy in dynamic systems. Two major ways of vibration damping can be 
Fig. 9 Post-processing of experimental data methodology
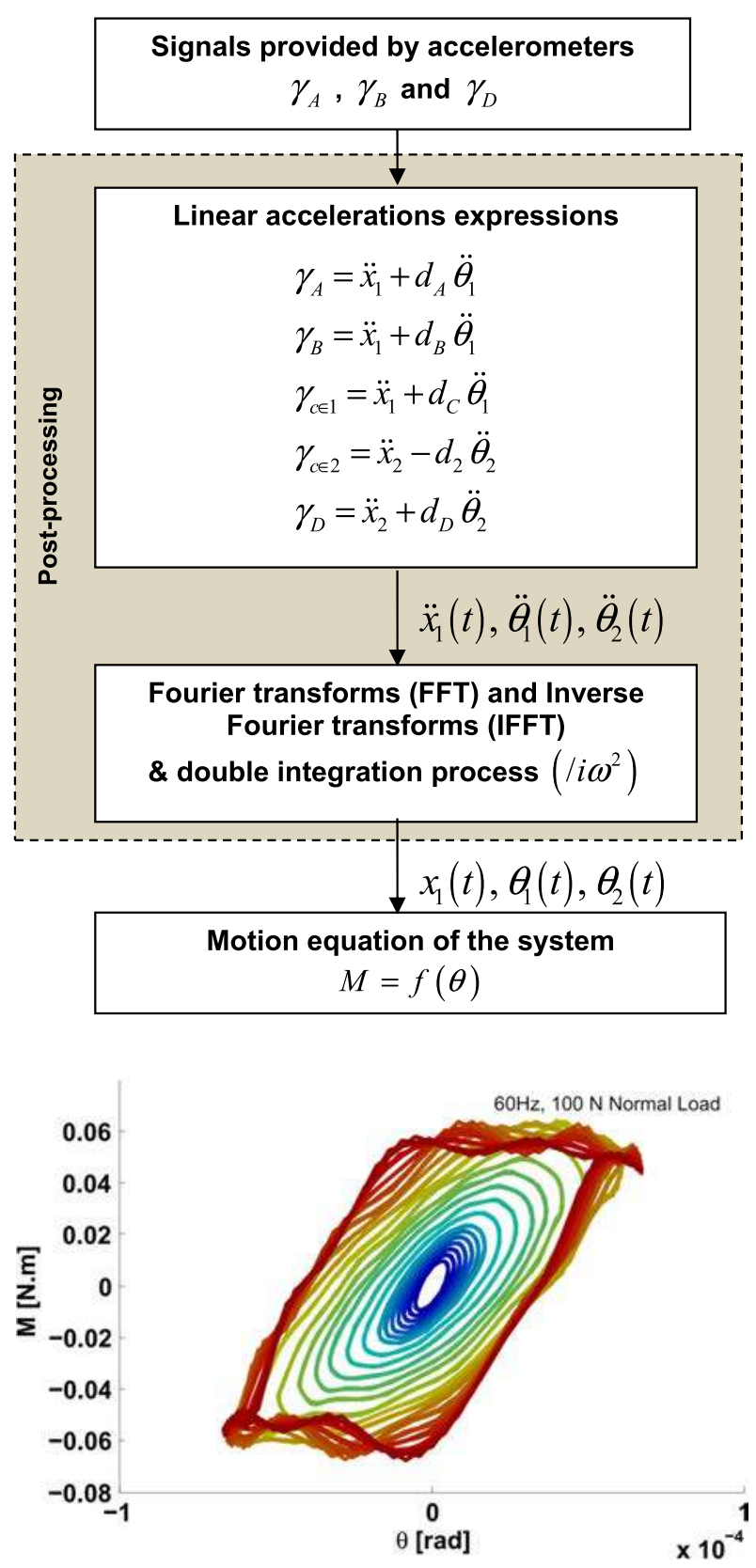

$$
\gamma_{A}, \gamma_{B} \text { and } \gamma_{D}
$$

$$
\begin{aligned}
& \gamma_{A}=\ddot{x}_{1}+d_{A} \ddot{\theta}_{1} \\
& \gamma_{B}=\ddot{x}_{1}+d_{B} \ddot{\theta}_{1} \\
& \gamma_{c \in 1}=\ddot{x}_{1}+d_{C} \ddot{\theta}_{1} \\
& \gamma_{c \in 2}=\ddot{x}_{2}-d_{2} \ddot{\theta}_{2} \\
& \gamma_{D}=\ddot{x}_{2}+d_{D} \ddot{\theta}_{2}
\end{aligned}
$$

Fig. 10 Experimental hysteresis plots. Torque as a function of angular displacement 
Fig. 11 Generalized Maxwell model

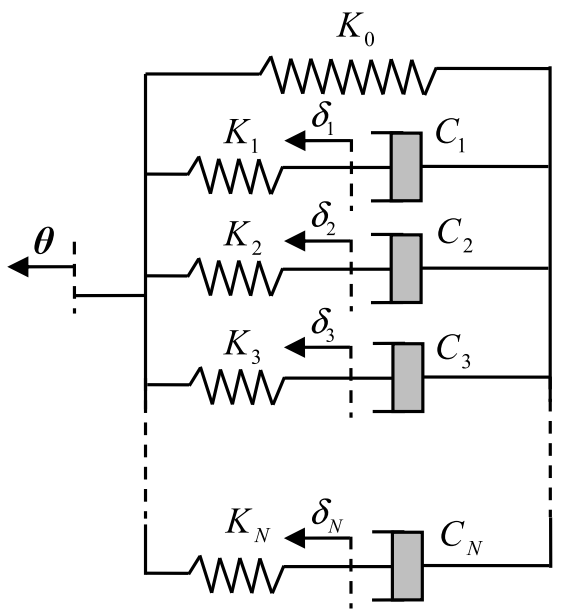

elastomeric joint subjected to harmonic rotational loading. A rheo-tribological model is developed by combining a rheological model and dry friction model, and extended taking into account dependence of the torque and the angular displacement (rotation).

The mechanical properties of a viscoelastic material can be represented by a Generalized Maxwell Model (GMM) which consists of a spring and several Maxwell cells (a spring and dashpot in series) assembled in parallel, see Fig. 11. GMM is equivalent to a complex modulus approach (Hu and Wereley 2007). It can describe relaxation which does not occur at a single time, but in a set of times. Due to Maxwell cells of different lengths with shorter ones contributing less than longer ones, there is a varying time distribution. Although the GMM cannot identify the creep process after a constant stress is applied to a material specimen, it can successfully characterize stiffness and damping during steady-state harmonic excitation (Popov and Geike 2007). Therefore, it is the simplest approach to describe the nonlinear behavior of elastomeric materials based on a Generalized Maxwell Model.

For the elastomeric joint subjected to harmonic angular displacement $\theta(t)$, its response is the sum of the spring feedback $M_{0}(t)$ added to each cell reaction, denoted $M(t)$ :

$$
M(t)=M_{0}(t)+\sum_{i=1}^{N} M_{i}(t) .
$$

The rheological formulation of the dynamic stiffness of GMM is

$$
Z(\omega)=K_{0}+\sum_{i=1}^{N} \frac{j \omega K_{i} C_{i}}{K_{i}+j \omega C_{i}}
$$

where $K_{0}$ is the stiffness taken at $\omega=0$, i.e. $t=+\infty, K_{i}$ is the stiffness of the $i$ th spring and $C_{i}$ is the damping of the $i$ th dashpot, $i \in[1 . . N]$.

Reducing Eq. (22) to the same denominator and grouping monomials gives the dynamic stiffness of linear GMM expressed as the ratio of two polynomials of the same degree $N$ (number of Maxwell cells) (Renaud et al. 2011). This formulation of transfer function is also used in automation, namely, Oustaloup (1991) provided a model using poles and zeros 


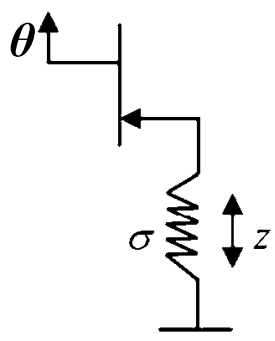

formulation (PZF).

$$
Z(\omega)=K_{0} \prod_{i=1}^{N}\left(\frac{1+\left(j \omega / \omega_{z, i}\right)}{1+\left(j \omega / \omega_{p, i}\right)}\right)
$$

$\omega_{z, i}$ and $\omega_{p, i}$ are respectively the zero and the pole of the $i$ th pole-zero couple, $i \in[1 . . N]$. This operator called by Oustaloup (1991) "CRONE regulator" facilitates considerably the treatment and the parametric identification of the polynomial ratio by expressing it in product form.

Several models have been studied in order to describe the dynamic friction observed for elastomeric samples. Most dynamic models of frictional behavior are built with internal state variables of kinematic type; however, these variables cannot be identified as real physical displacements (De Wit et al. 1995; Lampaert et al. 2002; Segalman 2005, 2006). The first model of this kind was proposed by (Dahl 1968) within frictional behavior was explained with an analogy for the stress-strain property for materials. Dahl compared this with the spring like elastic material behavior, occurring in the bonding forces between the two surfaces, Fig. 12. Therefore, Dahl model is used to model dynamic friction appearing at the elastomeric joint interfaces.

Dahl's model is mathematically very well formulated to be integrated in ODEs such as those which govern vibrations. These equations can be solved numerically in time domain (Dahl 1968; De Wit et al. 1995) or analytically (Dahl 1968).

Dahl proposed to model pre-displacement and hysteresis using the following expressions:

$$
\left\{\begin{array}{l}
\frac{d z}{d t}=\dot{\theta}\left|1-\frac{M}{M_{\max }} \operatorname{sign}(\dot{\theta})\right|^{\alpha} \operatorname{sign}\left(1-\frac{M}{M_{\max }} \operatorname{sign}(\dot{\theta})\right) \\
M(t)=\sigma z(t)
\end{array}\right.
$$

where $M$ is the frictional torque, $z$ is the internal displacement state variable, $\sigma$ is homogeneous with stiffness, $M_{\max }$ is the maximum torque reached, and $\alpha \in \mathrm{R}_{+}^{*}$ is a constant parameter which represents hysteresis torque-angular displacement curve, $\theta$ is the relative angular displacement, and $\dot{\theta}$ is the relative angular velocity.

From the viewpoint of modeling friction, Dahl's model can be considered as a nonlinear rheological model (Jrad et al. 2013a, 2013b; Dion et al. 2013). Moreover, Dahl model simulates nonlinear frictional behavior without frequency information; whereas, GMM captures the linear behavior of the elastomer under frequency excitation.

Thus, in this work, for combining the Dahl model and GMM it will be assumed that the torque generated by the frictional spring of Dahl model, $M(t)=\sigma z(t)$ is viscoelastic, so that $\sigma$ will be modeled by GMM. Hence, during the imposed displacement $\theta(t)$, the frictional spring will stretch to $z(t)$ which will excite the GMM. 
Fig. 13 Identification using hysteresis curves

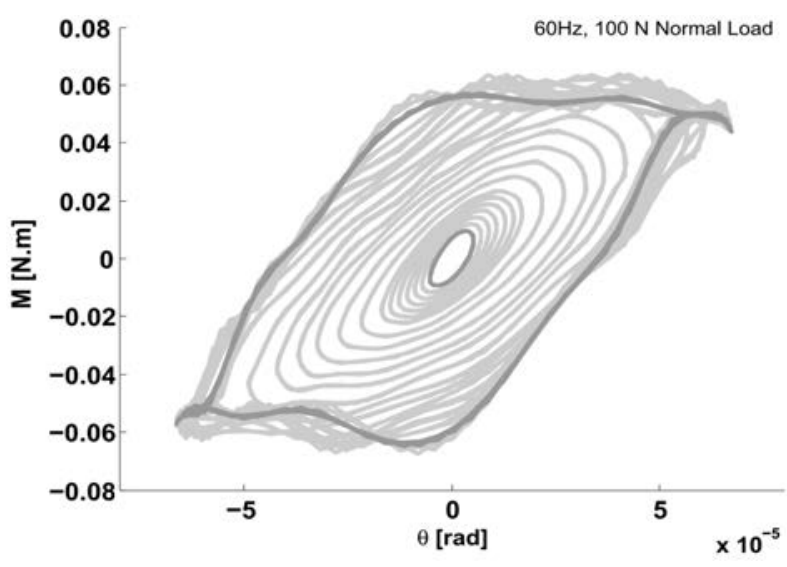

Basing in this approach, a rheo-tribological model is developed by coupling GMM and Dahl model taking into account dependence of the torque and the angular displacement (rotation):

$$
\left\{\begin{array}{l}
\dot{z}(t)=\dot{\theta}(t)\left|1-\frac{M(t)}{M_{\max }} \operatorname{sign}(\dot{\theta}(t))\right|^{\alpha} \operatorname{sign}\left(1-\frac{M(t)}{M_{\max }} \operatorname{sign}(\dot{\theta}(t))\right) \\
M(t)=K_{0} z(t)+\sum_{i=1}^{N} K_{i}\left(z(t)-\delta_{i}(t)\right) \\
K_{i}\left(z(t)-\delta_{i}(t)\right)=C_{i} \dot{\delta}_{i}(t) \\
M_{\max }=\mu M_{N}
\end{array}\right.
$$

where $\mu$ is the coefficient of sliding friction and $M_{N}$ is the torque due to normal preload.

\subsection{Parametric identification techniques of the rheo-tribological model}

The parametric identification is based on the subsequent model (Eq. (25)). This model needs to be previously identified with an experimental test. In that way, this phenomenological model could not be considered as a predictive model. Experimental tests are performed with periodic signals and allow identifying model parameters. In this section, the parametric identification method has been built to provide accuracy and can be automatically executed for a broad-based measurement campaign.

Hysteresis loops of Torque-Angular displacement involve details which enable identification of parameters of the proposed model. Techniques are proposed to identify viscoelastic parameters $K_{0}, K_{i}$ and $C_{i}$ and friction parameters $\mu$ and $\alpha$.

The quasi vertical part of the curve represents the system stiffness. The horizontal part of the curve is the significant behavior of the slip as shown in Fig. 13. Most parametric models (Dahl 1968; De Wit et al. 1995; Almajid 2004; Segalman 2005, 2006; and Thaijaroen and Harrison 2010) can be identified with this kind of curves.

Figure 14 shows torque (twisting moment) versus angular displacement (rotation) as a function of stiffness; the dashed lines with a mostly vertical direction define stiffness. Indeed, the dynamic stiffness is given by the slope of the hysteresis loop. 


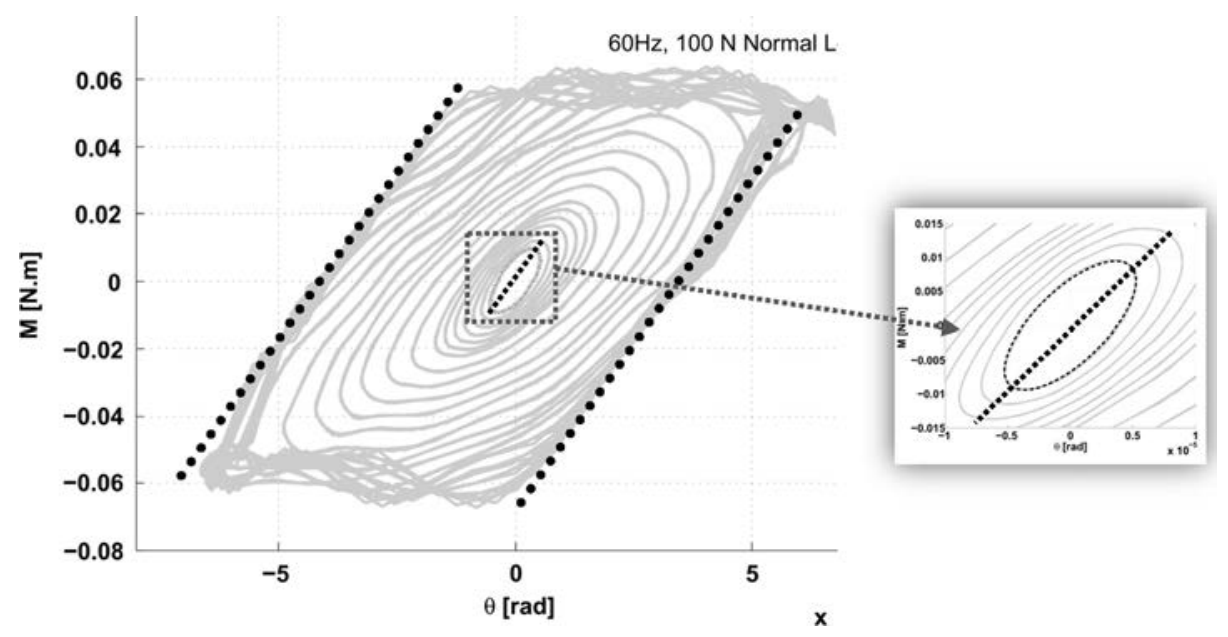

Fig. $14 K_{0}$ parameter identification

The first cycles of the diagram show pure ellipsoid curves which correspond to linear viscoelastic behavior. Thus, $K_{0}$ identified at the beginning within displacement is small and its value is estimated to be the slope of the major axis of an ellipse (in the Fourier sense) which coincides with one selected cycle of the first cycles as illustrated in Fig. 14.

Once we have determined $K_{0}$, the identification of $K_{i}$ and $C_{i}$ is built on hypothesis proposed by Renaud et al. (2011) who proposed expressions that allow quantifying $K_{i}$ and $C_{i}$ parameters of GMM using PZF (Poles-Zeros Formulation).

Considering $N$ Maxwell cells, $K_{i}$ and $C_{i}$ coefficients are given by the following equations after computing poles and zeros adequate to the studied frequency range:

$$
\left\{\begin{array}{l}
K_{i}=K_{0} \prod_{h=1}^{N}\left(\frac{\omega_{p, h}}{\omega_{z, h}}\right)\left(\frac{\omega_{p, i}-\omega_{z, h}}{\omega_{p, i}+\omega_{p, h}\left(\delta_{i h}-1\right)}\right) \\
C_{i}=\frac{K_{i}}{\omega_{p, i}}
\end{array}\right.
$$

The method used to determine the poles and zeros is analogous to the approach proposed by Oustaloup (1991) which main idea leads to consider the angle phase equal to $\pi / 2$ between zero and pole of the same order and null elsewhere (Fig. 15). The resulting phase angle in the considered frequency range is then estimated by averaging the phase angle computed between the first zero and the last pole (Renaud et al. 2011).

It will be assumed that the ratio between two consecutive zeros is constant and equal to the ratio between two consecutive poles in order to obtain a constant phase between two consecutive zeros, two constants $\chi$ and $\gamma$ are then obtained by the following equations:

$$
\left\{\begin{array}{l}
\ln (\chi)=\phi \frac{\ln \left(f_{2}\right)-\ln \left(f_{1}\right)}{\phi+\frac{\pi}{2}(N-1)} \\
\ln (\gamma)=\left(\frac{\pi}{2}-\phi\right) \frac{\ln \left(f_{2}\right)-\ln \left(f_{1}\right)}{\phi+\frac{\pi}{2}(N-1)}
\end{array}\right.
$$




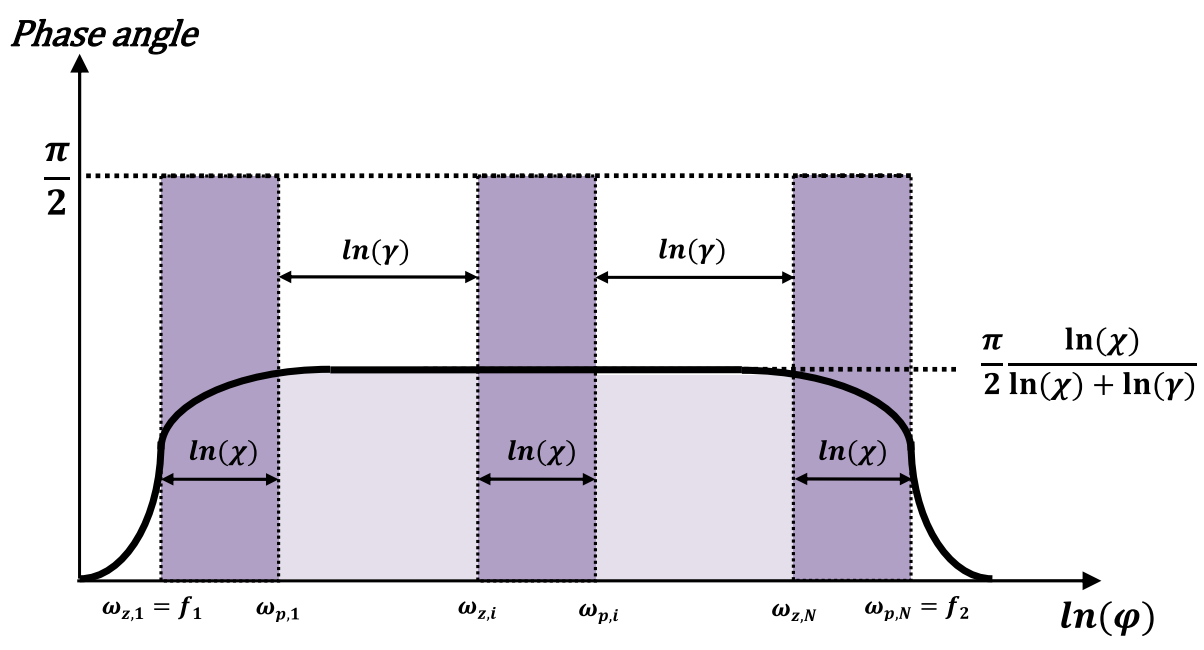

Fig. 15 Constant phase angle approach

where $f_{1}$ and $f_{2}$ are respectively the upper and lower bounds of the frequency domain. $\phi$ is the estimated mean phase angle for all tests.

It will be considered that the first zero coincides with $f_{1}$ and the last pole with $f_{2}$, as shown in Fig. 15, using this assumption $\chi$ and $\gamma$ can be determined. The identification of $K_{i}$ and $C_{i}$ can be performed using a ratio of two polynomial functions defined by zeros and poles. Initially, the zeros are determined from the first zero, then, the poles are computed from the last pole:

$$
\left\{\begin{array}{l}
\omega_{z, i+1}=\omega_{z, i} \chi \gamma \\
\omega_{p, i}=\frac{\omega_{p, i+1}}{\chi \gamma}
\end{array}\right.
$$

The relationship gathering zeros and poles is given by Eq. (29), so that all poles and zeros are estimated and $K_{i}$ and $C_{i}$ can be predicted:

$$
\omega_{p, i}=\chi \omega_{z, i} .
$$

The prediction of vibration levels is directly linked to the parameters of friction models (Jrad et al. 2013a, 2013b). The friction coefficient $\mu$ is defined by the horizontal tangent when the torque $M$ reaches its maximum as shown in Fig. 16. Hence, the friction coefficient is identified for the dynamic torsion test with static preload $\mathrm{P}$ using the given expression:

$$
\mu=\frac{M_{\text {max }_{-} \tan }}{P \ell}
$$

with $\ell$ being the thickness of the beam.

Table 1 shows estimated friction coefficient and maximum torque reached corresponding to the different tested preloads.

We consider then that the identified friction coefficient is constant and its value is $\mu=$ 0.3 .

The identification of $\alpha$ is based on the work of Peyret et al. (2010) who showed that there is an accurate identification between loading phase of contact between two surfaces defined 
Table 1 Estimated friction coefficient

\begin{tabular}{lllll}
\hline Preload $P[\mathrm{~N}]$ & 45 & 100 & 150 & 500 \\
\hline Maximum tangent torque $M_{\text {max_tan }}[\mathrm{Nm}]$ & 0.026 & 0.063 & 0.09 & 0.31 \\
Friction coefficient $\mu$ & 0.29 & 0.315 & 0.3 & 0.31 \\
\hline
\end{tabular}

Fig. 16 Friction coefficient identification

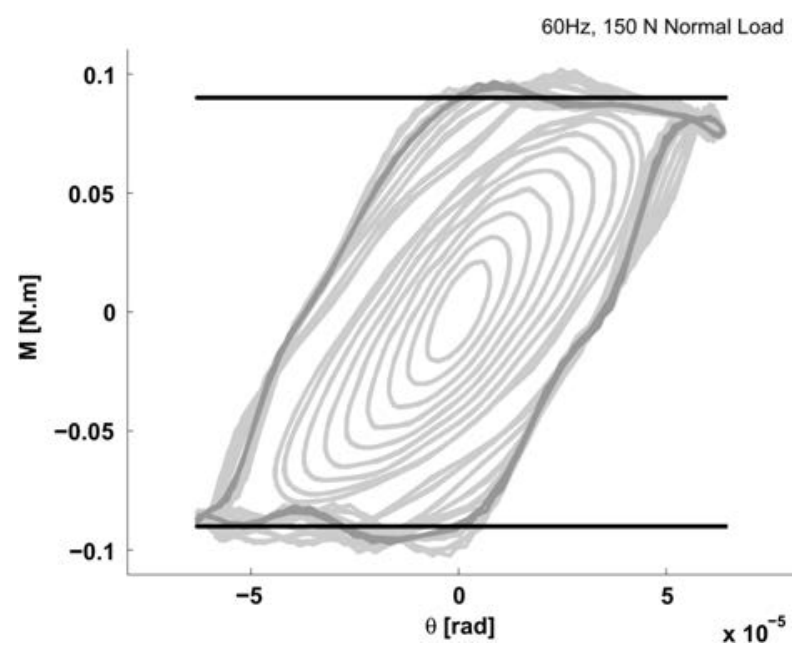

by Mindlin (1949) and parameters of Dahl model, in particular the constant parameter $\alpha$ which represents the shape of the hysteresis loop. Parametric analyses performed in this study with several excitation amplitudes and preloads have shown that $\alpha$ can be set at $1 / 3$ which is in concordance with Peyret et al. (2010) results.

Considering $N=4$ Maxwell cells, the previous identification method aims to reduce the number of 11 parameters ( 9 viscoelastic parameters and 2 friction parameters) to 3 independent and recognizable parameters that can be identified with only one test.

The proposed identification method is robust and has been applied for a very large number of tests with several frequencies and normal loads.

\section{Results and validation}

After the model parameters were determined, the output torque was predicted using known angular displacement input data, and then the torque-angular displacement hysteresis curves were reconstructed. In this section, in order to assess the model's capability in describing elastomer behavior under the complex loading conditions the reconstructed hysteresis curves and test data were compared for different displacement amplitudes.

Figure 17 shows comparison of the reconstructed hysteresis cycles of torque-angular displacement using the hysteresis model and the experimental data in the amplitude range of $\left[-6,5 \cdot 10^{-5} ; 6,5 \cdot 10^{-5}\right]$ rad at $60 \mathrm{~Hz}$ and under $100 \mathrm{~N}$ of normal preloading.

It can be noticed that simulated hysteresis matches the experimental data very well. Moreover, using the identified friction parameters, a torque-angular displacement hysteresis due to friction damping is reconstructed. It is known that energy dissipation due to the damp- 
Fig. 17 Measured and simulated torque-angular displacement curves overlayed one above the other

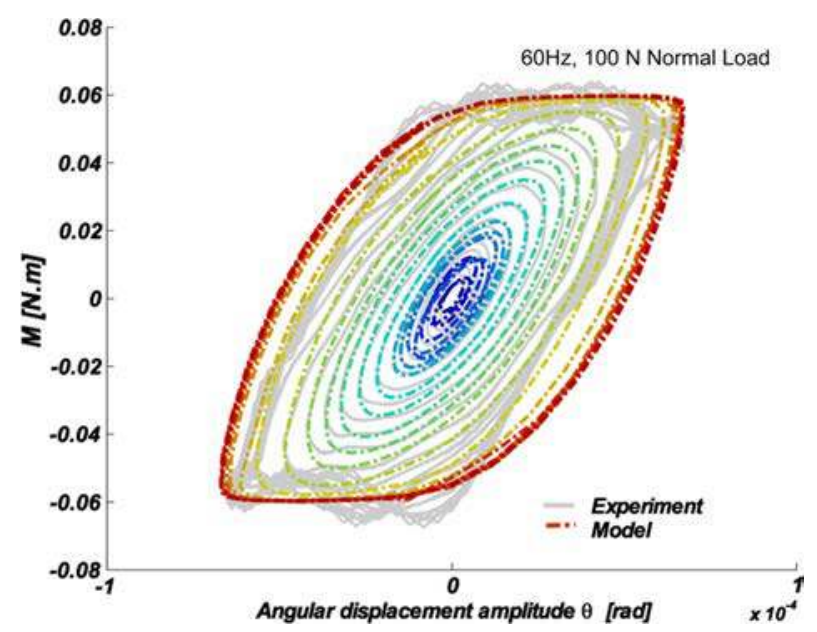

ing corresponds to the enclosed area inside the torque-angular displacement hysteresis loop. The area enclosed by the total torque-angular displacement hysteresis loop is equal to the area enclosed by the damping hysteresis. This proves that the nonlinear damping equation's system (Eq. (25)) precisely describes the energy dissipation while the elastomer specimen is under harmonic loading.

It can also be seen that at low amplitudes $\left[-8,5 \cdot 10^{-6} ; 8,5 \cdot 10^{-6}\right] \mathrm{rad}$, the experimental hysteresis plots are nearly elliptical in shape, while for higher amplitudes (starting from $\left[-4 \cdot 10^{-5} ; 4 \cdot 10^{-5}\right] \mathrm{rad}$ ) there is a deviation from this elliptical behavior. It is shown that the rheo-tribological model performs well in the moderate amplitude range, except it overpredicts the inner loop in some cases. However, the response under harmonic loading with varying amplitude is generally well correlated with model predictions.

Comparisons between measures and simulations of the torque-angular displacement curve are conducted for the different frequencies, preloads and angular displacement amplitudes and the quality of fitting between simulations and measurements in Fig. 17 is similar for most of the carried out tests. As a result, modeling results can accurately capture the hysteresis behavior exhibited by the elastomer. Indeed, compared to the elastomer hysteresis models developed by Snyder et al. (2001) and Hu and Wereley (2007), the current nonlinear model more accurately captures nonlinear torque-displacement time histories under harmonic loading.

Furthermore, the scope of this work is to predict damping caused not only by inherent elastomeric material but also frictional damping with the aim of providing a model capable of describing the energy losses and the damping ratio.

In this part, we expect to evaluate dissipated energy in order to estimate the damping ratio.

It is well known that the area enclosed by the loading and unloading curves of hysteresis loops corresponds to the energy dissipated $E_{d}$ as heat during cycles by both inner and frictional damping. Figure 18 represents the evolution of the dissipated energy versus the amplitude of the angular rotation $\theta$ under a normal load $100 \mathrm{~N}$. The shape of the curve highlights the nonlinear dissipative behavior of the elastomeric joint under torsion harmonic loading; indeed, the dissipated energy depends strongly on the angular amplitude of rotation. 
Fig. 18 Progression of dissipated energy as a function of the amplitude of angular displacement

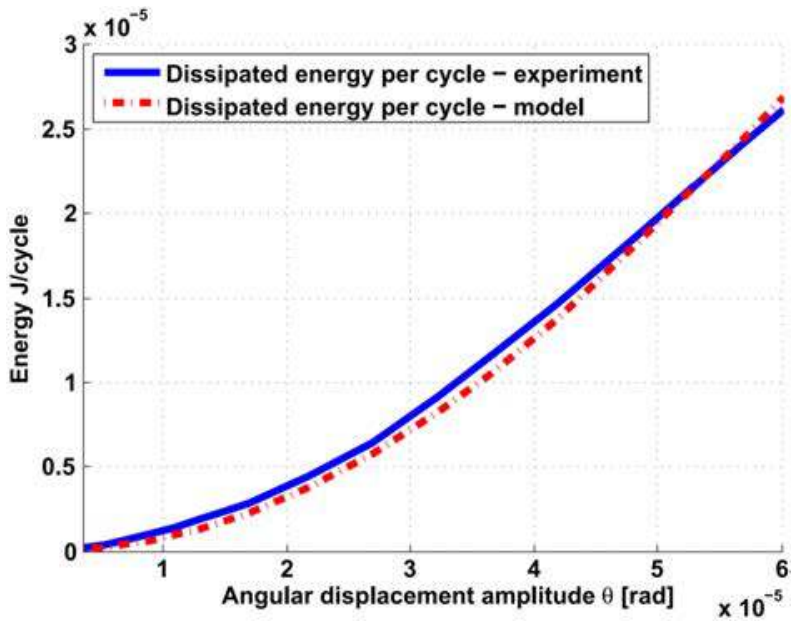

Fig. 19 Progression of damping ratio as a function of the amplitude of angular displacement

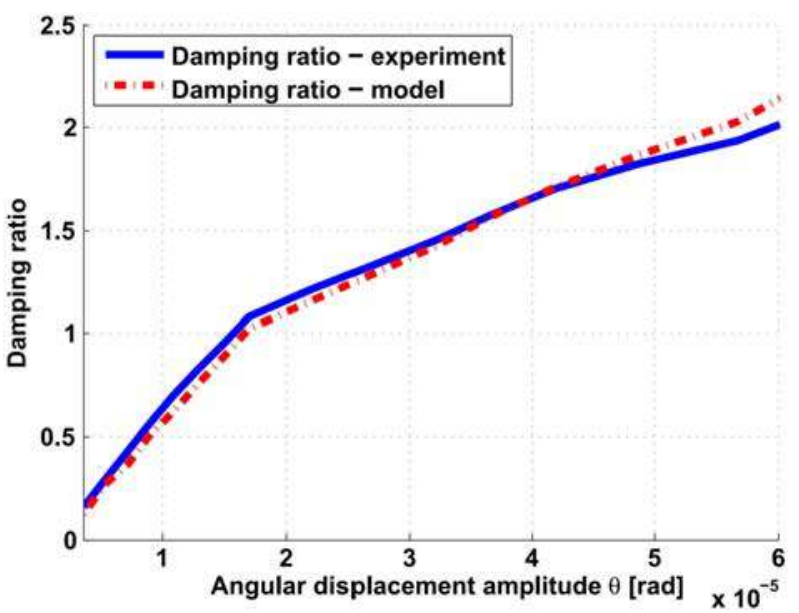

The damping ratio $\xi$ can be defined by the ratio between the dissipated energy $E_{d}$ and the reference energy $E_{r}$, (Jrad et al. 2013a, 2013b):

$$
\xi=\frac{4}{\pi} \frac{E_{d}}{E_{r}}
$$

where the reference energy $E_{r}$ for periodic signals is given by the following equation:

$$
E_{r}=4 \theta_{\max } M_{\max } .
$$

Here $\theta_{\max }$ is the amplitude of angular displacement.

The evolution of damping ratio $\xi$ as a function of angular displacement $\theta$ is presented in Fig. 19. It can be clearly observed that the damping ratio increases as the angular displacement increases. Therefore, we can say that the damping ratio is strongly dependent on the amplitude of the angular displacement which induces the nonlinear effects well shown in Fig. 18, since the form of the curve is parabolic. 
The dissipated energy and damping ratio determined by the proposed model is also compared to the experimental result. As shown in Figs. 18 and 19, the predicted dissipated energy and damping ratio using the model have the same amplitude dependent trend as the experimental result.

It can be concluded that the rheo-tribological can precisely describe the characteristics of the hysteresis loops and predict dissipated energy and damping ratio under slowly-varying amplitude of angular displacement.

The fidelity of the model was verified by the good correlation between simulations and the experimental results.

\section{Conclusion}

In a complex mechanism, the level of vibration strongly depends on its dissipation in the connected parts. This paper presents theoretical and experimental study on an assembled structure with a rotational elastomeric joint. A test bench was designed for improving the accuracy of measurements of nonlinear dissipative behaviors of the frictional interfaces of the elastomeric rotational joint and for quantifying the energy dissipated in order to compute the damping ratio at the interfaces under harmonic rotational loading. A new approach to post-processing experimental signals based on Lagrangian formalism, which allows accurately measuring forces and torques only with accelerometers, is proposed in order to identify dissipated energy in the rotational elastomeric joint. Measurement techniques and signal processing methods highlight excellent concordance between both direct measurements and parametric identification. A rheo-tribological model is a time-domain modeling approach to capture nonlinear behavior of the rotational elastomeric joint. The proposed model is able to accurately reconstruct the nonlinear hysteresis behavior exhibited by the elastomer and to precisely predict dissipated energy and damping ratio under slowly-varying amplitude of angular displacement which correlated very well with the corresponding experimental data. This model formulated as a state space model by coupling Generalized Maxwell and Dahl models has the advantage that it could easily be implemented into dynamic system models. Although only a one-dimensional elastomeric model is described in this paper, the rheotribological model can also be extended into a three-dimensional form such that it can be implemented easily into a finite element analysis for a complex elastomeric damper configuration.

\section{References}

Almajid, A.: Harmonic response of a structure mounted on an isolator modeled with a hysteretic operator: experiments and prediction. J. Sound Vib. 277(1-2), 391-403 (2004)

Barbosa, F., Farage, M.: A finite element model for sandwich viscoelastic beams: experimental and numerical assessment. J. Sound Vib. 317(1-2), 91-111 (2008)

Cantournet, S., Desmorat, R., Besson, J.: Mullins effect and cyclic stress softening of filled elastomers by internal sliding and friction thermodynamics model. Int. J. Solids Struct. 46(11-12), 2255-2264 (2009)

Castello, D., Rochinha, F., Roitman, N., Magluta, C.: Constitutive parameter estimation of a viscoelastic model with internal variables. Mech. Syst. Signal Process. 22(8), 1840-1857 (2008)

Coveney, V.A., Johnson, D.E., Turner, D.M.: A triboelastic model for the cyclic mechanical behavior of filled vulcanizates. Rubber Chem. Technol. 68(4), 660-670 (1995)

Dahl, P.R.: A solid friction model, The Aerospace Corporation, El-Secundo, TOR-158(H3107-18), California, 1968

De Wit, C.C., Olsson, H., Astrom, K.J., Lischinsky, P.: A new model for control of systems with friction. IEEE Trans. Autom. Control 40(3), 419-425 (1995) 
Dion, J.L., Chevallier, G., Penas, O., Renaud, F.: A new multicontact tribometer for deterministic dynamic friction identification. Wear 300(1), 126-135 (2013)

Gandhi, F., Chopra, I.: A time-domain non-linear viscoelastic damper model. Smart Mater. Struct. 5(5), 517528 (1996)

Hu, W., Wereley, N.M.: Distributed rate-dependent elastoslide model for elastomeric lag dampers. J. Aircr. 44(6), 1972-1984 (2007)

Huynh, A., Argoul, P., Point, N., Dion, J.L.: Rheological models using fractional derivatives for linear viscoelastic materials: application to identification of the behavior of elastomers. In: Proceedings of VISHNO-13th Vibrations Shocks and Noise Conferences, Lyon, France (2002)

Jrad, H., Dion, J.-L., Renaud, F., Tawfiq, I., Haddar, M.: Experimental characterization, modeling and parametric identification of the nonlinear dynamic behavior of viscoelastic components. Eur. J. Mech. A, Solids 42, 176-187 (2013a)

Jrad, H., Renaud, F., Dion, J.-L., Tawfiq, I., Haddar, M.: Experimental characterization, modeling and parametric identification of the hysteretic friction behavior of viscoelastic joints. Int. J. Appl. Mech. 5(2), 1350018 (2013b) (1-22)

Lampaert, V., Swevers, J., Al-Bender, F.: Modification of the Leuven integrated friction model structure. IEEE Trans. Autom. Control 47(4), 683-687 (2002)

Lesieutre, G.A., Bianchini, E.: Time domain modeling of linear viscoelasticity using augmenting thermodynamic fields. In: Proceedings of the 34th AIAA/ASME/ASCE/AHS/ASC Structures, Structural Dynamics and Materials Conference. La, Jolla, CA, 1993 (1993)

Lesieutre, G.A., Mingori, D.L.: Finite element modeling of frequency-dependent material damping using augmenting thermodynamic fields. J. Guid. Control Dyn. 13(6), 1040-1050 (1990)

Luo, W., Hu, X., Wang, C., Li, Q.: Frequency- and strain-amplitude-dependent dynamical mechanical properties and hysteresis loss of CB-filled vulcanized natural rubber. Int. J. Mech. Sci. 52(2), 168-174 (2010)

Liu, M., Hoo Fatt, M.S.: A constitutive equation for filled rubber under cyclic loading. Int. J. Non-Linear Mech. 46(2), 446-456 (2011)

Mindlin, R.D.: Compliance of elastic bodies in contact. J. Appl. Mech. 16, 259-268 (1949)

Oustaloup, A.: La commande CRONE: commande robuste d'ordre non entier. Hermés (1991)

Panda, B., Mychalowycz, E., Tarzanin, F.J.: Application of passive dampers to modern helicopters. Smart Mater. Struct. 5(5), 509-516 (1996)

Park, S.W.: Analytical modeling of viscoelastic dampers for structural and vibration control. Int. J. Solids Struct. 38(44-45), 8065-8092 (2001)

Peyret, N., Dion, J.L., Chevallier, G., Argoul, P.: Microslip induced damping in planar contact under constant and uniform normal stress. Int. J. Appl. Mech. 2(2), 281-304 (2010)

Popov, V.L., Geike, T.: A new constitutive model of rubber. Tribol. Int. 40(6), 1012-1016 (2007)

Ramrakhyani, D.S., Lesieutre, G.A., Smith, E.C.: Modeling of elastomeric materials using nonlinear fractional derivative and continuously yielding friction elements. Int. J. Solids Struct. 41(14), 3929-3948 (2004)

Renaud, F., Dion, J.L., Chevallier, G., Tawfiq, I., Lemaire, R.: A new identification method of viscoelastic behavior: application to the generalized Maxwell model. Mech. Syst. Signal Process. 25(3), 991-1010 (2011)

Rendek, M., Lion, A.: Amplitude dependence of filler-reinforced rubber: experiments, constitutive modelling and FEM - implementation. Int. J. Solids Struct. 47, 2918-2936 (2010)

Saad, P., Al Majid, A., Thouverez, F., Dufour, R.: Equivalent rheological and restoring force models for predicting the harmonic response of elastomer specimens. J. Sound Vib. 290(3), 619-639 (2006)

Segalman, D.J.: A four-parameter Iwan model for lap-type joints. Int. J. Appl. Mech. 72(5), 752-760 (2005)

Segalman, D.J.: Modelling joint friction in structural dynamics. Struct. Control Health Monit. 13, 430-453 (2006)

Skouvaklis, G., Blackford, J.R., Koutsos, V.: Friction of rubber on ice: a new machine, influence of rubber properties and sliding parameters. Tribol. Int. 49, 44-52 (2012)

Snyder, R.A., Kamath, G.M., and Wereley, N.M.: Characterization and analysis of magnetorheological damper behavior under sinusoidal loading. AIAA J. 39(7), 1240-1253 (2001)

Strganac, T.W.: An experiment and analytical methodology to characterize nonlinear elastomeric lag dampers. In: Proceeding of the 38th AIAA/ASME/ASCE/AHS/ASC Structures, Structural Dynamics, and Material Conference, Orlando, Florida, April (1997)

Thaijaroen, W., Harrison, A.J.L.: Nonlinear dynamic modelling of rubber isolators using six parameters based on parabolic spring, spring pot, and smooth-slip friction element. Polym. Test. 29(7), 857-865 (2010)

Tarzanin, F.J., Panda, B.: Development and application of nonlinear elastomeric and hydraulic lag damper models. In: Proceedings of the 36th AIAA/ASME/ASCE/AHS/ASC Structures, Structural Dynamics, and Materials Conference, New Orleans, Louisiana, April (1995) 